\title{
The significance of serum oligoclonal bands in neurological diseases
}

\author{
A Zeman, B McLean, G Keir, R Luxton, M Sharief, E Thompson
}

\begin{abstract}
The presence of oligoclonal bands (OCBs) of immunoglobulin G (IgG) in CSF provides evidence for the occurrence of a humoral immune response, but it is not always appreciated that the oligoclonal IgG may have originated in the serum. To determine the diagnostic significance of serum OCBs 146 patients with serum OCBs were identified among 1874 patients with suspected neurological disorders (7.6\%). Clear diagnoses had been made in 112 of these patients: in 56 identical CSF and serum bands were present, revealing a systemic immune response, while in $\mathbf{4 6}$ additional unique CSF bands indicated that intrathecal IgG synthesis was also occurring. In the first group neoplasia and peripheral neuropathies accounted for over $50 \%$ of the diagnoses, infections and systemic inflammatory disorders for $32 \%$, and multiple sclerosis was diagnosed in only one case. These figures contrast considerably with those reported for patients with CSF OCBs alone. Diagnoses in the second group of patients, with unique CSF OCBs in addition to serum OCBs, resembled those among patients with CSF OCBs alone. Examining CSF and serum in parallel for OCBs of IgG provides more diagnostic information than examining CSF alone, and the latter is potentially misleading.
\end{abstract}

(F Neurol Neurosurg Psychiatry 1993;56:32-35)

The demonstration of OCBs of IgG in the CSF provides valuable evidence of the occurrence of a humoral immune response. ${ }^{1-3}$ However, from the point of view of clinical diagnosis, OCBs are a non-specific finding: a wide range of pathologies, in particular infection, inflammation and neoplasia, may provoke such an immune response. ${ }^{3}$ Sensitive modern immunochemical methods ${ }^{4}$ demonstrate OCBs of immunoglobulin $G$ in the serum as well as in the CSF in an appreciable proportion of cases: comparison of the response in CSF and serum in a given patient would offer an opportunity to narrow down the differential diagnosis of OCBs if it turned out that distribution of OCBs between CSF and serum had distinct diagnostic implications. In fact, this is the case, and the main aim of this paper is to provide a "user's guide" to the interpretation of the presence of serum oligoclonal bands.

\section{Patients and methods}

1) Isoelectric focusing

CSF and serum were examined for OCBs of immunoglobulin $G$ by isoelectric focusing, followed by passive protein transfer to a nitrocellulose membrane and immunostaining of the protein attached to the membrane for immunoglobulin $G$ by a double antibody method. This technique has been described fully in a previous paper. ${ }^{4}$ In brief, it involves application of a 2.5 microlitres of sample (diluted 1:400 in the case of serum) to an agarose gel containing synthetic ampholytes (molecules bearing variable net charge). Following the application of an electric field across the gel, ampholytes and proteins migrate to a position in the gel determined by their isoelectric point (the $\mathrm{pH}$ at which they bear no net charge). This achieves a good separation of IgG clones, as individual clones tend to differ in isoelectric point. The proteins in the gel are then transferred to a nitrocellulose membrane, to which they stick passively, by laying the membrane over the gel and applying a weight. Clones of IgG are visualised by incubation of the membrane with an antibody to human IgG, followed by a second antibody directed against the first and coupled to an enzyme (horseradish peroxidase) which can be visualised chemically. By this method a monoclone of IgG generates several bands: these, however, have a very characteristic appearance, so that there is generally no difficulty in distinguishing bands indicative of a single clone from those indicative of two or more clones. The method is very tolerant of variations in the CSF IgG concentration, and yields satisfactory results over a 50 fold range, from applications of 20 to $1200 \mathrm{ng}$ of IgG. ${ }^{4}$

2) Classification of isoelectric focusing patterns An oligoclonal response is defined as the presence of two or more distinct antibody clones. These antibody clones are visible because they stand out against the background of polyclonal antibody as a result of the relatively intense stimulation of a corresponding number of clones of lymphocytes responsible for their production. Three patterns of oligoclonal response in CSF and serum are distinguished: 1) patients with at least two clones in CSF, but none in serum, have an exclusively intrathecal oligoclonal response: we refer to this pattern as "intrathecal-only" (symbolised in the figure by $a+)$; 2) patients with at least two clones which are identical in CSF and serum have evidence of a systemic oligoclonal response. We refer to this pattern as "systemic- 
Figure Three sets of paired serum (s) and CSF (c) samples illustrate the patterns of oligoclonal response $\left(+,>,{ }^{\star}\right)$ defined in the text.

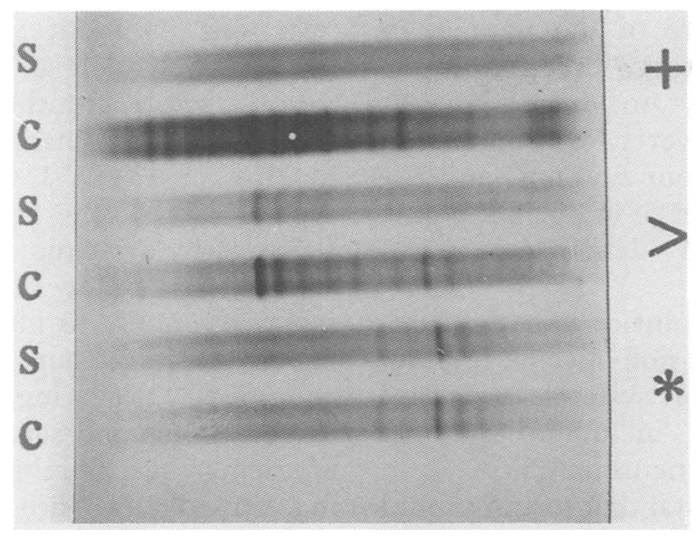

only" (symbolised in the figure by $a^{\star}$ ); 3 ) patients with at least one clone in serum and at least one clone unique to CSF, have both intrathecal and systemic responses. We refer to this pattern as "intrathecal-systemic" (symbolised in the figure by a $>$ ).

Isoelectric focusing patterns were reported by four of the authors (AZ, BM, ET, GK) without knowledge of any clinical details.

\section{3) Patients and diagnoses}

A total of $1874 \mathrm{CSF}$ and serum pairs were examined of which $146(7 \cdot 8 \%)$ contained serum OCBs. Of these 146, 88 patients belonged to a group of consecutive samples from 10 hospitals examined between January and March 1991; the remaining 58 belonged to a consecutive series of samples from inpatients at the National Hospital for Neurology examined between 1985-88. Some details from the second group of patients have been reported previously. ${ }^{3}$ Diagnoses were obtained either by studying case notes or from discharge summaries. Adequate clinical details were not available for 10 patients and these were not included in further analyses. Diagnoses were sorted into 10 major groups corresponding to broad diagnostic categories which are commonly used in clinical practice: infections, systemic inflammatory disorders including connective tissue disorders and sarcoidosis, paraneoplastic syndromes, neoplasia excluding paraneoplastic syndromes, Guillain-Barré syndrome, other peripheral neuropathies, multiple sclerosis, vascular disorders, and degenerative disorders. One hundred and two patients could be classified confidently; the remainder were undiagnosed.

\section{4) Statistical analysis}

The significance of interactions between pathological processes and isoelectric focusing patterns were analysed using the Chi square test for $2 \times 2$ tables. Fisher's exact test was applied if any expected cell values were less than 5 .

\section{Results}

Table 1 shows the diagnoses of patients with the "systemic-only" and "intrathecal-systemic" responses.

The main findings were: 1) peripheral neuropathies were diagnosed commonly among
Table 1 Frequency of pathological processes responsible for isoelectric focusing patterns (percentages in brackets)

\begin{tabular}{lcc}
\hline & $\star$ & $>$ \\
\hline Infections & $8(14)$ & $13(29)$ \\
"Inflammations" & $10(18)$ & $4(9)$ \\
Paraneoplastic & $3(5)$ & $2(4)$ \\
Neoplastic & $9(16)$ & $1(2)$ \\
GBS & $9(16)$ & $0(0)$ \\
Other PN & $10(18)$ & $0(0)$ \\
Multiple sclerosis & $1(2)$ & $26(57)$ \\
Vascular & $3(5)$ & $0(0)$ \\
Degenerative & $3(5)$ & $0(0)$ \\
Totals & $56(99)$ & $46(100)$ \\
\hline
\end{tabular}

GBS = Guillain-Barré syndrome, other $\mathrm{PN}=$ other varieties of peripheral neuropathy. ${ }^{\star}$ refers to isoelectric focusing patterns peripheral neuropathy. "refers to isoelectric focusing pattern with identical CSF and serum OCBs, $>$ to patterns with at least one clone identical in CSF and serum
unique CSF clone. See text for further details.

patients with identical CSF and serum OCBs ("systemic-only" response) but did not occur among patients displaying the "intrathecalsystemic" response $(p<0.0001) ; 2)$ similarly, neoplastic disorders were common among patients with identical CSF and serum bands but were rarely diagnosed among patients with the "intrathecal-systemic" response $(p<0.05)$. Where the latter response was seen in association with neoplasia a paraneoplastic syndrome or lymphocytic CNS tumour was responsible; 3) multiple sclerosis was the most frequent single cause of the "intrathecal-systemic" response but occurred in only 1 patient with identical OCBs in CSF and serum $(p<0.0001) ; 4)$ however, an examination of the results for the entire patient group with serum OCBs, including both patients with the "systemic-only" and with the "intrathecalsystemic" response, revealed that infectious and inflammatory disorders were found among these patients as often as multiple sclerosis.

\section{Discussion}

A wide variety of methods have been used to demonstrate immunoglobulin synthesis within the CNS. A qualitative method, isoelectric focusing, is known to give a more sensitive indication of the occurrence of an intrathecal humoral immune response than do quantitative methods: ${ }^{3}$ the presence of clonal antibodies in the CSF which are absent from the serum provides very strong evidence of an intrathecal immune response.

The occurrence of serum OCBs is not widely appreciated, and little information is available on their significance. Identical CSF and serum $\mathrm{OCBs}$ provide evidence of systemic immune activation but not of intrathecal synthesis: in this case the presence of OCBs in the $\mathrm{CSF}$ is explained by their passive movement from the serum into the CSF across the bloodbrain barrier which offers only a relative obstacle to serum proteins. ${ }^{5}$ (In theory additional intrathecal synthesis might sometimes be contributing in these cases but quantitative measurements would be necessary to determine this). In patients in whom serum OCBs are accompanied by additional OCBs unique to CSF there is evidence of both systemic and intrathecal synthesis of immunoglobulin.

The proportions of results falling in the various categories we have described are 
dependent on the nature of the population sampled by our laboratory, and cannot be extrapolated confidently to very different patient populations. However, certain general principles emerge from our findings, and the results reinforce a number of important points about the nature and demonstration of OCBs.

First, from the standpoint of everyday clinical diagnosis, they are a non-specific finding, occurring in infections, in systemic and local inflammatory disorders including multiple sclerosis, in peripheral neuropathies and in neoplasia. Second, it is vital that serum should be examined in parallel with CSF to establish whether CSF OCBs are the result of intrathecal or of systemic synthesis, or of a combination of the two, and thus to narrow down the diagnostic implications of a positive result. This recommendation has been made in the past, ${ }^{12}$ but is often ignored.

Turning to the pathologies which give rise to OCBs, the most frequent single indication for the immunological analysis of CSF is the investigation of suspected multiple sclerosis. Our previous findings, typical of many others, ${ }^{6}$ have indicated that multiple sclerosis characteristically gives rise to intrathecal OCBs without a detectable serum response. A substantial proportion of patients with multiple sclerosis, however, do display serum bands, ${ }^{7}$ which are fainter and fewer than their counterparts in CSF: the results reported here suggest that multiple sclerosis is the most common single cause of such a mixed "intrathecal-systemic" response. This is of theoretical interest, as it points to systemic immune activation. We are at present investigating associations between the presence of serum bands in patients with multiple sclerosis and clinical features, for example the question of whether the presence of serum bands might be related to infective triggers of relapse.

Identical OCBs in CSF and serum are very rarely seen in multiple sclerosis of any kind and, like a negative result, should lead to reconsideration of the diagnosis. Failure to compare CSF and serum patterns could be seriously misleading where, for example, the differential diagnosis lies between a myelopathy and a peripheral neuropathy.

Infections can give rise to any of the three patterns of OCBs: however, it was striking from examination of the specific diagnoses among our patients that OCBs identical in $\mathrm{CSF}$ and serum tended to result from systemic

Table 2 Occurrence of OCB patterns in major categories of disease

\begin{tabular}{lllll}
\hline & + & $>$ & $\star$ & specificity? \\
\hline Infection & yes (esp CNS) & yes (esp CNS) & yes (esp syst) & yes $^{1013}$ \\
Inflammation & yes (esp CNS) & yes (esp CNS) & yes (esp syst) & yes 14 $^{17}$ \\
Paraneoplastic & yes & yes & yes & yes \\
Neoplastic & rare & rare & yes & no \\
GBS & no & no & yes & no \\
Other neuropathies & no & no & yes & no \\
Multiple sclerosis & yes & yes & no & no \\
Vascular & no & no & rare & no \\
Degenerative & no & no & rare & no
\end{tabular}

Syst = systemic, GBS = Guillain-Barre syndrome, "Specificity?" refers to the question of whether, in at least some instances, the antigenic specificity of the OCBs has been determined. + refers to isoelectric focusing patterns with OCBs in CSF only: for explanation of ${ }^{\star}$ and - see figure and isext. infections without primary neurological involvement, such as septicaemia and endocarditis, while intrathecal synthesis occurred where a pathogen had penetrated the CNS, as in HIV or HTLV-1 infection, or in neurosyphilis.

Similarly, systemic inflammatory disorders can give rise to any of the three patterns, but most commonly to identical CSF and serum bands. Among our patients inflammatory conditions triggering this "systemic-only" response included sarcoidosis, systemic lupus erythematosus, rheumatoid arthritis, ankylosing spondylitis with uveitis and primary anti-phospholipid antibody syndrome. Inflammatory disorders giving rise to the "intrathecal-systemic" response included sarcoidosis, the stiff man syndrome and Eale's disease.

If neoplastic disorders are associated with OCBs these are generally identical in serum and CSF. Paraneoplastic syndromes are an exception as they can also give rise to intrathecal synthesis. Rarely, lymphocytic tumours of the CNS re also associated with intrathecal immunoglobulin synthesis. It is interesting that systemic neoplasia should, on this evidence, appear to provoke an systemic humoral immune response quite commonly.

Peripheral neuropathies accounted for $34 \%$ of cases with OCBs identical in CSF and serum in our series. There may be several explanations for the systemic immune response to which this finding bears witness: Guillain-Barré syndrome is often preceded by an infection which might provoke such a response, and OCBs have been reported in this condition in the past; ${ }^{8}$ neuropathies such as those due to vitamin B12 deficiency in pernicious anaemia, diagnosed in one of the patients in the present series, occur in a context of systemic autoimmunity; the occasional presence of OCBs in inherited and metabolic neuropathies suggests other, less straightforward, processes are at work.

Table 2 summarises our experience of the distribution of OCB patterns among the pathological groups. Although it is of theoretical interest that a number of conditions causing intrathecal IgG synthesis also give rise to serum OCBs, the diagnostic implications of the "intathecal-only" and the "intrathecalsystemic" responses are broadly similar; by contrast, the finding of identical CSF and serum OCBs, the "systemic-only" response, raises the possibility of a variety of systemic disorders. The mechanisms giving rise to OCBs are complex, and other groups have, for example, reported an incidence of intrathecal synthesis of oligoclonal Immunoglobulin G in as many as $11 \%$ of patients with cerebrovascular diseases. ${ }^{9}$ We believe that differences in patient selection and in immunochemical methods, especially in the choice of ampholytes, ${ }^{4}$ underly the discrepancy between these results and ours.

While we have emphasised that the presence of OCBs is a non-specific and ambiguous finding, this is, in a sense, the source of its value: the presence of OCBs of immunoglobu- 
lin $G$ demonstrates the occurrence of a humoral response in disorders in which we are unsure of the nature of the antigen exciting the response, or indeed of whether the response is directly triggered by an antigen at all. It is, however, now possible to probe the antigenic specificity of OCBs using antigen specific immunoblotting techniques: thus bands directed against infectious agents (for example HIV, ${ }^{10}$ HTLV1, ${ }^{11}$ Varicella zoster, ${ }^{12}$ poliovirus $^{13}$ ), host molecules (such as, glutamic acid decarboxylase in the stiff man syndrome $\mathrm{e}^{14}$ and a variety of neuronal proteins in paraneoplastic syndromes ${ }^{15}{ }^{16}$ ) and diagnostic reagents (for example, Kveim antigen in sarcoidosis ${ }^{17}$ ) have all been demonstrated. Like OCBs of "total" immunoglobulin $G$, antigen specific bands may be found in CSF alone or both in CSF and serum, and their distribution may have diagnostic value.

In conclusion, the differential diagnosis of OCBs in neurological disease is wide and includes infections, systemic inflammatory disorders, multiple sclerosis, peripheral neuropathies and neoplasia. Examination of CSF and serum specimens in parallel is valuable. Multiple sclerosis is sometimes associated with an oligoclonal serum response in addition to the typical intrathecal oligoclonal response. Peripheral neuropathies and neoplasia often give rise to OCBs common to CSF and serum, indicating a systemic humoral response with "leakage" of antibody into the CSF. Infections and inflammatory disorders may be associated with any of the three patterns of oligoclonal response: in these conditions unique CSF bands suggest direct involvement of the CNS. Considering patients with serum bands as a group, infectious and systemic inflammatory conditions are responsible for this laboratory finding as often as multiple sclerosis.

We thank consultants at the following hospitals for supplying samples to our laboratory and for allowing us to examine patient records: The National Hospitals for Neurology and Neurosurgery, University College Hospital, The Middlesex
Hospital, Guy's Hospital, St Thomas' Hospital, The Royal Free Hospital, The Hammersmith Hospital, Addenbrooke's Hospital, The Ipswich Hospital, The Humana Hospital. We thank the staff of the Special Chemistry Laboratory at the Institute of Neurology for their painstaking work, and Mr Peter Sacares for statistical advice.

1 Fishman RA. Cerebrospinal fluid in disorders of the nervous system. Philadelphia: WB Saunders, 1980

2 Link $H$. Cerebrospinal fluid in immunological CNS diseases. In: Aarli JA, Behan WMH, Behan PO, eds. Clinical neuroimmunology. Oxford: Blackstreets, 1987.

3 McLean BN, Luxton RW, Thompson EJ. A study of immunoglobulin $G$ in the cerebrospinal fluid of 1007 patients with suspected neurological diseases using isopatients with suspected neurological diseases using iso-
electric focusing and the log-IgG index. Brain 1990;113:1269-89.

4 Keir G, Luxton RW, Thompson EJ. Isoelectric focusing of cerebrospinal fluid IgG: an annotated update. Ann clin biochem 1990;27:436-43.

5 Thompson EJ. The CSF proteins: a biochemical approach. Amsterdam: Elsevier, 1988

6 Tourtellotte WW. The cerebrospinal fluid in multiple sclerosis. Handbook of clinical neurology, vol 3 (47): demyelinating diseases. Amsterdam: Elsevier, 1985:35-46.

7 Laurenzi MA, Mavra M, Kam-Hansen S, Link H. Oligoclonal IgG and free light chains in multiple sclerosis demonstrated by thin-layer polyacrylamide gel isoelectric focusing and immunofixation. Ann Neurol 1980;8: focusin

8 Link H. Demonstration of oligoclonal immunoglobulin G in Guillain-Barré syndrome. Acta Neutol Scand 1975;52: $111-20$

9 Kostulas VK, Link H, Lefvert A-K. Principles for demonstration and interpretation based on findings in 1114 neurological patients. Arch Neurol 1987;44:1041-4.

10 Bukasa K S-S, Sindic CJM, Bodeus M, Burtonboy G Laterre C, Sonnet J. Anti-HIV antibodies in the CSF of AIDS patients: a serological and immunoblotting study. $\mathcal{F}$ Neurol Neurosurg Psychiatry 1988;51:1063-8.

11 McLean BN, Rudge P, Thompson EJ. Viral specific IgG and IgM in the cerebrospinal fluid of patients with tropical IgM in the cerebrospinal fluid of patients with

12 Schadlich H-J, Nekic M, Jeske J, Karbe K. Intrathecal humoral immune reaction in zoster infections. $\mathcal{f}$ Neurol Sci 1991;103:101-4.

13 Sharief $M K$, Hentges $R$, Ciardi $M$. Intrathecal immune response in patients with the post-polio syndrome. $N$ Engl f Med 1991;325:749-55.

14 Solimena M, Folli F, Denis-Donini S, et al. Autoantibodies to glutamic acid decarboxylase in a patient with stiff-man syndrome, epilepsy, and type 1 diabetes mellitus. $N$ Engl f Med 1988;318:1012-20.

15 Anderson NE, Rosenblum MK, Posner JB. Paraneoplastic cerebellar degeneration: clinical-immunological correlations. Ann Neurol 1988;24:559-67.

16 Anderson NE, Rosenblum MK, Graus F, Wiley RG, Posner JB. Autoantibodies in paraneoplastic syndromes assoJB. Autoantibodies in paraneoplastic syndromes asso-
ciated with small-cell lung cancer. Neurology 1988;38: ciated with $1391-8$.

17 McLean BN, Mitchell DN, Thompson EJ. Local synthesis of specific IgG in the cerebrospinal fluid of patients with neurosarcoidosis detected by antigen immunoblotting using Kveim material. $\mathcal{f}$ Neurol Sci 1990;99:165-75. 